\title{
Évolution de la couche d'ozone sous l'effet du protocole de Montréal et du changement climatique
}

\author{
Sophie Godin-Beekmann \\ Laboratoire atmosphères, milieux, observations spatiales (LATMOS), \\ Centre national de la recherche scientifique (CNRS), \\ Institut Pierre-Simon Laplace (IPSL), \\ Université de Versailles Saint-Quentin-en-Yvelines \\ 11 boulevard d'Alembert - 78280 Guyancourt Cedex
}

\section{Résumé}

La destruction, à partir du début des années 1980, de la couche d'ozone stratosphérique, par des constituants halogénés issus de l'industrie chimique, est considérée aujourd'hui comme la première atteinte des activités humaines à l'environnement atmosphérique global. En parallèle, le protocole de Montréal, signé en 1987 pour réguler l'émission des substances destructrices d'ozone, fait figure de pionnier dans le domaine de la réglementation mondiale destinée à protéger l'environnement terrestre. Il a notamment inspiré le protocole de Kyoto signé en 1992 pour réguler les émissions des gaz à effet de serre. Plus de deux décennies après la signature du protocole de Montréal, le présent article fait le point sur la réduction des émissions de gaz destructeurs d'ozone, sur l'état de la couche d'ozone et sur les prévisions quant à son rétablissement, compte tenu du changement climatique et de son influence sur le retour à l'équilibre de l'ozone. Le bénéfice additionnel du protocole de Montréal quant aux émissions de gaz à effet de serre est également évoqué. e problème de la destruction de la couche d'ozone et la signature du protocole de Montréal apparaissent aujourd'hui comme la success story de la politique environnementale à l'échelle planétaire. Plus connu et médiatisé sous le nom de « trou d'ozone », ce phénomène a suscité un immense intérêt public et une inquiétude légitime quant au devenir de la couche d'ozone à l'échelle globale. L'enjeu était d'importance puisque l'ozone est un gaz vital qui protège la vie sur Terre en filtrant le rayonnement UV-B solaire ${ }^{(1)}$. Ce rayonnement a un effet néfaste sur les organismes vivants car il altère l'ADN des cellules. Un accroissement du rayonnement UV-B est notamment dangereux pour la santé : il augmente les risques de cancer de la peau et l'occurrence des cataractes, et il affaiblit le système immunitaire.

Dans le milieu des années 1980, des chercheurs anglais et japonais font état d'une diminution très importante de l'ozone mesuré en Antarctique pendant le printemps austral, en octobre (Farman et al., 1985 ; Chubachi, 1984). L'ampleur de la destruction de l'ozone, restituée par les mesures satellitaires, se traduit par une réduction de plus de $50 \%$ de son contenu intégré sur une superficie atteignant celle du continent antarctique, et par une disparition quasi complète entre 16 et $20 \mathrm{~km}$ d'altitude. Ces mesures ont provoqué une réaction rapide de la communauté scientifique internationale. Quelques années seulement après la découverte du trou d'ozone polaire, les processus à l'origine de ce phénomène ont été identifiés, permettant d'incriminer le rôle de gaz halogénés produits industriellement, les chlorofluorocarbures (CFC) et les halons ${ }^{(2)}$. Un accord est alors rapidement intervenu au sein de la communauté internationale pour réguler la production de ces gaz, qui s'est traduit par la signature du protocole de Montréal en 1987.

Depuis la fin des années 1980, la couche d'ozone fait ainsi l'objet d'une attention particulière et d'importants moyens ont été mis en œuvre pour évaluer son amincissement à l'échelle globale. Avec l'arrêt des émissions des substances nocives pour l'ozone, on s'attend au rétablissement progressif de la couche d'ozone, prévu pour le milieu du XXI ${ }^{\mathrm{e}}$ siècle, le temps que la stratosphère évacue les gaz polluants injectés par les activités humaines. Plusieurs événements ont cependant jeté le trouble sur les prévisions de reconstitution de l'ozone stratosphérique. En 2002, un réchauffement majeur du vortex polaire antarctique au mois de septembre, phénomène jamais observé auparavant dans cette région, induit une réduction importante de la superficie du trou d'ozone. A contrario, en 2011, une destruction record d'ozone est observée en Arctique. Compte tenu

(1) Rayonnement de longueurs d'onde comprises entre $280 \mathrm{~nm}$ et $320 \mathrm{~nm}$.

(2) Les CFC sont composés d'atomes de carbone, de fluor et de chlore. Les CFC les plus importants sont le $\mathrm{CFC}-11\left(\mathrm{CClF}_{3}\right)$ et le $\mathrm{CFC}-12\left(\mathrm{CCl}_{2} \mathrm{~F}_{2}\right)$. Les halons sont composés d'atomes de carbone, de brome, de fluor et parfois de chlore (par exemple, le halon-1301: $\mathrm{CBrF}_{3}$ ). 
. + . / " .

\section{Abstract}

\section{The stratospheric ozone layer and the Montreal Protocol}

The destruction of the stratospheric ozone layer from the early 1980 s by halogen compounds originated from the chemical industry is considered the first global damage to the Earth's atmosphere by human activities. Likewise, the Montreal Protocol signed in 1987 in order to regulate ozone depleting substances is a pioneering agreement in the field of environmental regulation for the protection of the environment on the global scale. It has inspired the Kyoto Protocol for the reduction of greenhouse gases emission. More than twenty years after the Montreal Protocol, this article reviews the state of the ozone layer at global scale, the reduction of ozone depleting substances in the atmosphere, the previsions for the ozone recovery and the influence of global atmospheric change due to increased greenhouse gases emissions on this recovery. The double benefit of the Montreal Protocol on the emission of greenhouse gases is also described.

(1) S. Rowland, M. Molina et P. Crutzen ont obtenu le prix Nobel de physique en 1995 pour leurs travaux sur l'ozone stratosphérique. Paul Crutzen a mis en évidence, en 1970, le rôle des composés azotés sur l'équilibre chimique de l'ozone.

(2) Chimie faisant intervenir des composés en phase gazeuse avec des composés en phase liquide ou solide. de ces informations contradictoires, quelles sont les prévisions actuelles quant au rétablissement de la couche d'ozone ? Le changement climatique dû aux émissions de gaz à effet de serre peut-il perturber ce rétablissement ? Enfin, comment évaluer le succès du protocole de Montréal ? Cet article a pour objectif de faire le point sur les recherches actuelles menées sur ce sujet et sur les différentes hypothèses quant au retour à l'équilibre de l'ozone stratosphérique.

\section{Équilibre naturel de la couche d'ozone}

L'ozone $\left(\mathrm{O}_{3}\right)$ est un gaz très minoritaire dans l'atmosphère avec un rapport de mélange qui ne dépasse pas 10 à 12 molécules par million de molécules d'air (partie par million en volume ou ppmv). Son abondance est maximale dans la stratosphère, région atmosphérique située entre 10 et $50 \mathrm{~km}$ d'altitude. Dans les très basses couches atmosphériques, l'ozone, qui est un gaz fortement oxydant, peut être toxique si sa concentration est trop importante (pollution photo-oxydante ou smog). Dans la stratosphère, la production d'ozone est due à la photodissociation des molécules d'oxygène par le rayonnement solaire ultraviolet et à la recombinaison des atomes d'oxygène produits avec les molécules d'oxygène avoisinantes. Cette production est compensée par la destruction chimique de l'ozone, qui fait intervenir d'autres composés tels que les composés hydrogénés, azotés, chlorés et bromés, par le biais de cycles catalytiques. Reconstituant les composés réactifs initiaux destructeurs d'ozone, ces cycles de réactions chimiques permettent à des constituants, dont la concentration est mille fois inférieure à celle de l'ozone (quelques parties par milliard de molécules d'air ou ppbv), d'avoir une influence notable sur cet élément. Au-dessus de 25-30 km, l'ozone est en équilibre photochimique, c'est-à-dire qu'il s'adapte instantanément aux variations de production ou de perte chimique. En dessous de $30 \mathrm{~km}$, le rayonnement solaire est moins fort et la concentration d'ozone est essentiellement régie par les processus de transport atmosphérique, sauf au printemps polaire, en Antarctique où, en raison de l'augmentation de l'abondance des composés halogénés par les activités humaines, les processus chimiques de destruction dominent les processus de transport.

\section{La couche d'ozone perturbée : élucidation du trou d"ozone en Antarctique}

Lélucidation des mécanismes responsables de la destruction massive de l'ozone en Antarctique a radicalement modifié la vision antérieure des processus chimiques qui affectent l'ozone stratosphérique, uniquement fondée, à l'époque, sur des réactions chimiques en phase gazeuse. Comme l'indique le rapport d'évaluation international sur l'ozone stratosphérique de 1985, la théorie montrait alors que l'influence des composés chlorés sur l'évolution de l'ozone stratosphérique était maximale vers $40 \mathrm{~km}$ d'altitude (WMO, 1985), une altitude où la concentration de l'ozone est faible. Il en résultait que l'augmentation de ces composés dans l'atmosphère, déjà notée en 1974 par Rowland et Molina ${ }^{(1)}$, ne pouvait entraîner qu'une diminution de quelques pour cents de la couche d'ozone, compte tenu de la faible abondance de l'ozone à cette altitude. La mise en évidence d'une destruction importante de l'ozone stratosphérique vers $20 \mathrm{~km}$, la zone d'altitude où sa concentration est maximale, a alors suscité de nombreuses interrogations quant à la compréhension de l'équilibre de l'ozone. Dès 1986 est formulée l'hypothèse que des processus chimiques en phase hétérogène ${ }^{(2)}$, impliquant des composés chlorés, pourraient jouer un rôle dominant dans la destruction de l'ozone polaire (Solomon et al., 1986). Cette hypothèse est vérifiée lors d'une campagne de mesures organisée en 1987 par la NASA (National Aeronautics and Space Administration). Au cours de celle-ci, des mesures simultanées d'ozone et de monoxyde de chlore, par un instrument installé à bord d'un avion stratosphérique volant à $18 \mathrm{~km}$ d'altitude, ont démontré le lien direct entre l'augmentation des composés chlorés présents dans la stratosphère et la destruction d'ozone. En l'espace de quelques années, les coupables ont ainsi été identifiés et le scénario permettant d'expliquer l'apparition du trou d'ozone polaire a été élucidé. Les fautifs sont les chlorofluorocarbures et les halons dont la remarquable inertie chimique a suscité nombre d'applications industrielles, de l'isolation thermique à la climatisation, en passant par la propulsion des principes actifs dans les bombes aérosol. Principalement émis, à l'époque, par les pays développés, ces 
composés sont transportés par la circulation atmosphérique dans la stratosphère où ils sont décomposés par le rayonnement ultraviolet (UV) solaire. Le temps mis à atteindre les pôles par les constituants halogénés et leurs produits de décomposition est de 3 à 6 ans, suivant l'altitude. L'étude des mécanismes impliqués dans l'apparition du trou d'ozone a montré que les ingrédients nécessaires à la destruction de l'ozone stratosphérique pendant le printemps polaire sont les suivants :

- une forte augmentation de la concentration en composés halogénés dans la stratosphère. Les composés chlorés ont vu leur concentration multipliée par 5 tandis que celle des composés bromés a augmenté de $50 \%$, du fait des activités humaines ;

- des conditions de températures hivernales très basses dans la stratosphère antarctique et la formation de nuages stratosphériques polaires ;

- la réapparition du rayonnement solaire au-dessus du continent antarctique au début du printemps.

La disparition du rayonnement solaire au-dessus de l'Antarctique en hiver entraîne la formation du vortex polaire, une ceinture de vents d'ouest très intenses qui isole les masses d'air polaires des régions avoisinantes. La stratosphère polaire se refroidit fortement, jusqu'à atteindre des températures très basses (inférieures à $-85^{\circ} \mathrm{C}$ ), propices à la formation de nuages stratosphériques polaires composés de cristaux de glace ou de gouttelettes d'eau et d'acide nitrique. Comme la stratosphère est une région très sèche (le contenu en vapeur d'eau y est de l'ordre de quelques parties par million), les régions polaires sont les seules dans lesquelles des nuages peuvent se former à cette altitude. À la surface de ces nuages, des réactions chimiques en phase hétérogène transforment alors les produits de dissociation des CFC, tels que le nitrate de chlore $\left(\mathrm{ClONO}_{2}\right)$ et l'acide chlorhydrique $(\mathrm{HCl})$, en composés susceptibles de détruire l'ozone $\left(\mathrm{Cl}_{2}, \mathrm{ClOH}\right)$. C'est l'étape d'activation du chlore. Dès la réapparition du soleil au-dessus du pôle, ces composés forment à leur tour du monoxyde de chlore $(\mathrm{ClO})$ qui, sous l'action du rayonnement solaire, initie des cycles catalytiques de destruction d'ozone, dont les deux plus importants impliquent le dimère $\mathrm{ClOOCl}$ et le monoxyde de brome (BrO). La concentration de $\mathrm{ClO}$ est alors multipliée par 500 à l'intérieur du vortex polaire, et l'ozone est détruit au rythme de plusieurs pour cents par jour. Cela aboutit à une diminution de plus de $60 \%$ de la colonne totale d'ozone au mois d'octobre, avec une disparition quasi complète de l'ozone entre 14 et $20 \mathrm{~km}$.

\section{Destruction d'ozone stratosphérique dans les régions polaires}

Dans l'hémisphère sud, le trou d'ozone est devenu un phénomène saisonnier récurrent depuis les années 1980. La destruction d'ozone débute dès le début de l'hiver, dans les régions périphériques du vortex polaire, du fait de la présence continue du rayonnement solaire. Les processus de destruction s'accélèrent à partir de la mi-août dans l'ensemble du vortex au fur et à mesure que le soleil s'élève au-dessus de l'horizon, pour atteindre une destruction quasi totale de l'ozone dans la basse stratosphère à la fin septembre. Les masses d'air appauvries en ozone restent ensuite confinées à l'intérieur $\mathrm{du}$ vortex polaire jusqu'à la rupture de ce dernier, qui intervient de façon variable entre la fin octobre et début décembre. Le trou d'ozone atteint son maximum de superficie au début du mois d'octobre. Cette surface maximale a régulièrement augmenté au cours des années 1980 puis s'est stabilisée, dans la décennie suivante, autour de 20 à 25 millions de $\mathrm{km}^{2}$ (voir figure 1), sauf en 2002 où elle n'a pas

dépassé 12 millions de $\mathrm{km}^{2} \mathrm{du}$ fait de la perturbation du vortex polaire par un réchauffement majeur de la stratosphère (Allen et al., 2003). Généralement observés dans la stratosphère arctique, ces événements se traduisent par une inversion du gradient de température, en hiver, entre les moyennes latitudes et les pôles, accompagnée d'une inversion de la circulation zonale avec l'apparition de vents d'est qui rompent le vortex polaire. Les réchauffements stratosphériques polaires font disparaître les nuages stratosphériques et stoppent les processus de destruction d'ozone. Depuis les années 2000, la surface du trou d'ozone montre une variabilité accrue, avec un record de 30 millions de $\mathrm{km}^{2}$ atteint en 2006. En 2011, cette surface était de 25 millions de $\mathrm{km}^{2}$ (figure 2).

Figure 2 - Trou d'ozone en octobre 2011. http://ozonewatch.gsfc.nasa.gov/monthly/ monthly 2011-10 SH.html

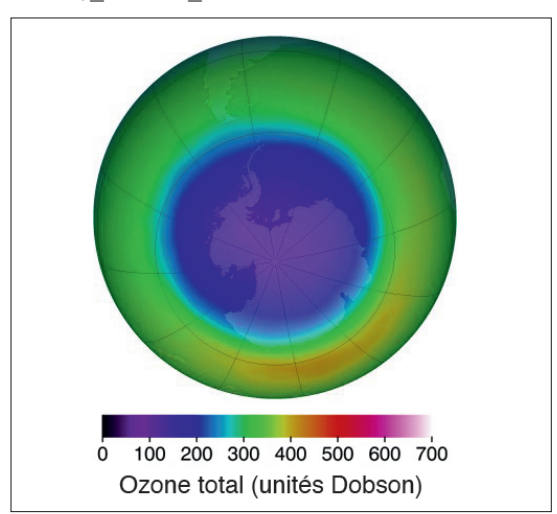

Figure 1 - Évolution du contenu intégré moyen d'ozone au-dessus du continent antarctique, en octobre, depuis les années 1970. Le trou d'ozone correspond à des valeurs inférieures à 220 unités Dobson (DU), soit les régions bleu foncé et mauves dans la figure (WMO, 2011).

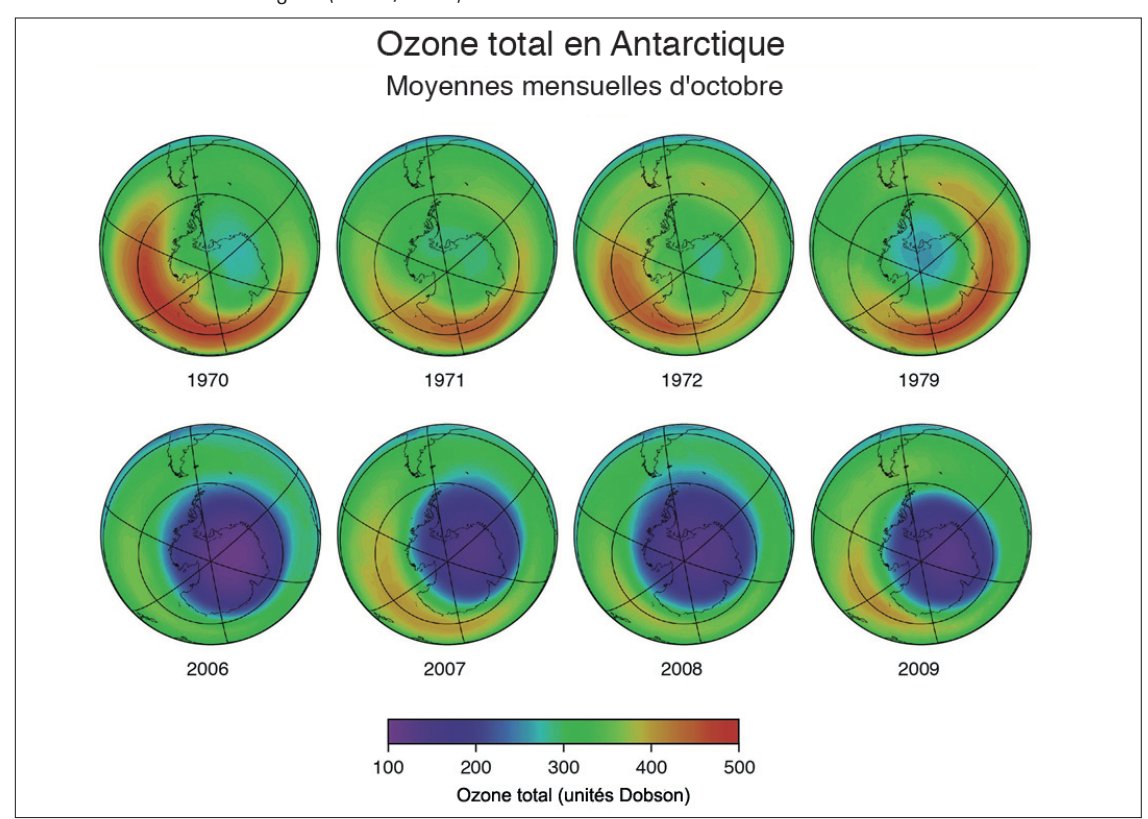


Après la découverte du trou d'ozone antarctique, la question s'est rapidement posée de l'éventualité d'un phénomène similaire dans la stratosphère arctique. De nombreuses campagnes de mesures ont alors été organisées par les États-Unis et l'Europe pour évaluer la destruction d'ozone dans cette région. Une différence essentielle entre les stratosphères antarctique et arctique est liée aux températures hivernales, beaucoup plus élevées dans le dernier cas. Cette dissymétrie s'explique par le fait que, dans l'hémisphère Nord, les continents remontent plus haut en latitude que dans l'hémisphère Sud et qu'ils occupent une surface plus importante. L'alternance de continents et d'océans et la présence de montagnes particulièrement hautes comme l'Himalaya génèrent davantage d'ondes atmosphériques à large échelle, qui se traduisent par la présence de zones de haute et de basse pression dans l'atmosphère. Certaines de ces ondes se propagent dans la stratosphère, déplacent le vortex polaire vers les latitudes plus basses et le réchauffent. Les conditions météorologiques sont ainsi beaucoup plus variables dans la stratosphère arctique, et la formation de nuages stratosphériques polaires est très différente d'une année sur l'autre. Certains hivers des années 1990 et l'hiver 1999/2000 ont toutefois été caractérisés par des conditions de température très basses, provoquant une destruction importante d'ozone. Pendant le printemps ont été observées des colonnes totales d'ozone inférieures de 20 à $30 \%$ aux valeurs mesurées

Figure 3 - Contenu intégré d'ozone mesuré par l'instrument satellitaire IASI en mars 2011.

http://www.ipsl.fr/Actualites/Actualites-scientifiques/ Diminution-record-d-ozone-au-pole-nord.

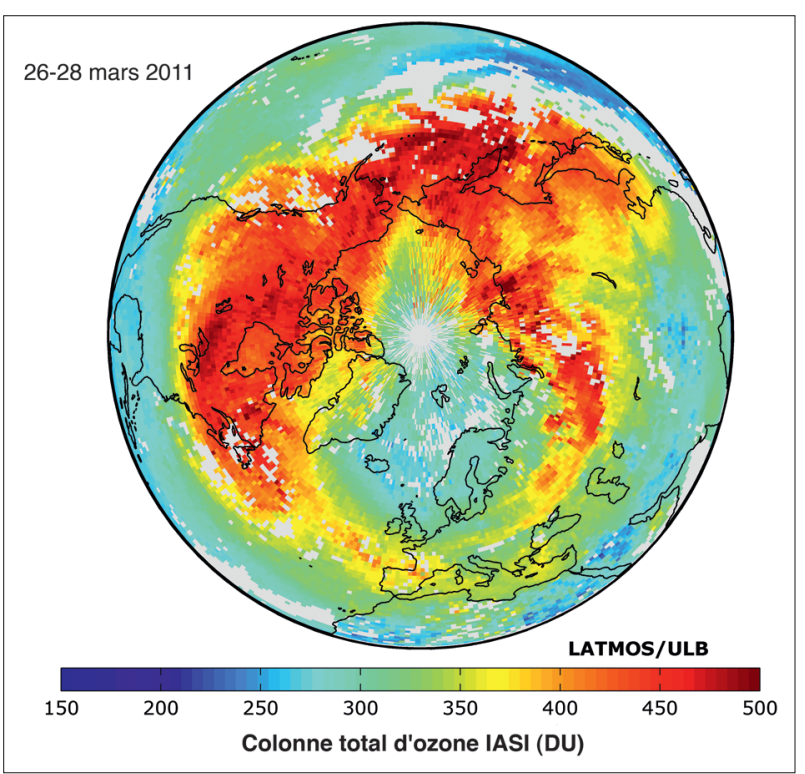

dans les années 1960-1970. Plus récemment, au cours de l'hiver arctique 2010/2011, une destruction record d'ozone a été détectée. Cette destruction, qui a atteint $40 \%$ en avril 2011, s'explique par un hiver très froid et persistant dans la stratosphère. Ces conditions exceptionnelles, s'apparentant à celles observées habituellement en Antarctique, ont conduit à une prolongation inhabituelle des processus de destruction d'ozone polaire jusqu'au printemps (Manney et al., 2011). La figure 3 montre les faibles valeurs d'ozone observées en mars 2011 par l'instrument IASI (Interféromètre atmosphérique de sondage infrarouge) embarqué sur le satellite européen METOP.

\section{Destruction d'ozone à l'échelle globale}

Depuis les années 1980, un important dispositif instrumental permettant des mesures au sol et satellitaires a été mis en œuvre pour surveiller l'évolution de la couche d'ozone. Ces mesures ont montré une diminution de l'ozone à l'échelle globale jusqu'à la fin des années 1990, variable suivant la latitude et la saison. L'éruption volcanique cataclysmique du mont Pinatubo en 1991, aux Philippines, a induit une réduction significative mais transitoire de l'ozone à l'échelle globale. Cette éruption a mis en lumière l'importance des processus de chimie hétérogène impliquant les composés chlorés et se produisant à la surface des aérosols volcaniques ${ }^{(1)}$. L'évolution de la distribution verticale d'ozone a été caractérisée, pendant la même période, par une diminution marquée, de l'ordre de $7 \%$ par décennie, dans deux régions distinctes, situées entre 15 et $20 \mathrm{~km}$ et autour de $40 \mathrm{~km}$ d'altitude. Dans cette dernière région, la déperdition d'ozone est due aux processus de chimie homogène impliquant les composés chlorés issus des CFC. Compte tenu de l'abondance plus importante de l'ozone dans la basse stratosphère, c'est la diminution dans cette zone d'atlitude qui a le plus fort impact sur l'évolution du contenu intégré d'ozone. Dans les régions de moyennes latitudes, la perte d'ozone est due à la dilution des masses d'air polaires appauvries en ozone à la suite de la rupture du vortex polaire, et aux processus chimiques in situ qui impliquent les composés halogénés (WMO, 1998).

\section{Le protocole de Montréal et ses amendements}

À la suite de la découverte du trou d'ozone antarctique, le protocole de Montréal a été signé en 1987 par 24 pays et mis en application en 1989. Il a actuellement été ratifié par plus de 190 pays (http://ozone.unep.org/new_site/en/ treaty_ratification_status.php). Le protocole et ses amendements successifs, signés entre 1990 et 2007 , ont conduit, dès 1996, à l'arrêt quasi total, dans les pays développés, de la production des composés chlorés et bromés responsables de la destruction de l'ozone stratosphérique. Les principaux composés réglementés sont les chlorofluorocarbures CFC-11 et CFC-12, le tétrachlorure de carbone, les halons 1211 et 1301 et le bromure de méthyle. Dans l'atmosphère, les composés chlorés sont environ cent fois plus abondants que les composés bromés, mais le pouvoir de destruction de l'ozone de ces derniers par les cycles catalytiques est près de 45 fois plus élevé. Ces différents produits ont été remplacés dans l'industrie chimique par les hydrochlorofluorocarbures (HCFC), beaucoup moins nocifs pour la couche d'ozone. Du fait de la présence d'atomes d'hydrogène, ces gaz sont en effet dissociés dans la troposphère et seule une petite fraction atteint la stratosphère. Outre ces réglementations, le protocole souligne l'importance de la surveillance de la couche d'ozone à l'échelle mondiale à partir de différents instruments de mesure, embarqués sur satellite ou installés au sol. Il prévoit également la mise au point régulière, par la communauté scientifique internationale, de rapports d'évaluation sur l'état de la couche d'ozone. Le dernier rapport a été publié en 2011 (WMO, 2011).

(1) Gouttelettes liquides composées d'eau et d'acide sulfurique et injectées, de façon massive, dans la stratosphère par les éruptions volcaniques cataclysmiques telles que celle du mont Pinatubo. 


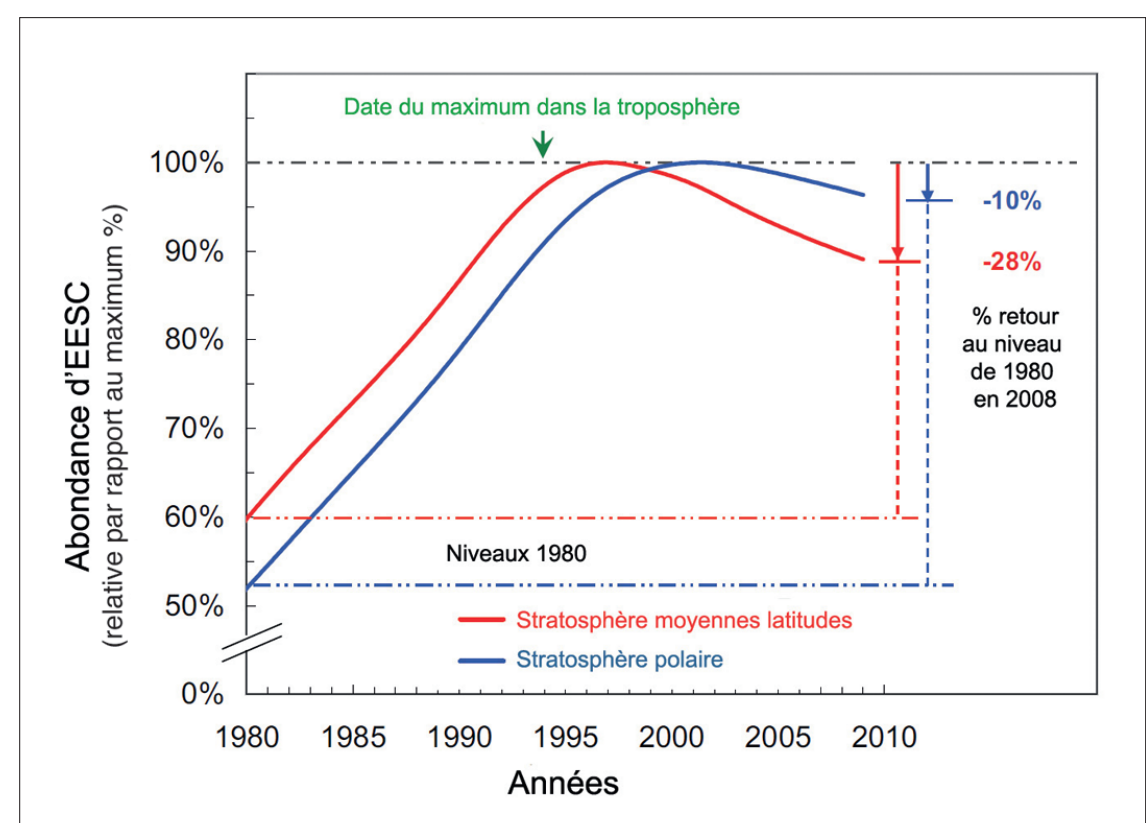

Figure 4 - Évolution temporelle de la charge effective en chlore dans la stratosphère (EESC), consécutive au protocole de Montréal, dans les régions de moyennes latitudes (courbe rouge) et dans les régions polaires (courbe bleue). Adapté de WMO (2011).

L'application du protocole de Montréal et de ses amendements a permis de ralentir puis d'inverser l'augmentation de la concentration des substances nocives pour l'ozone dans la stratosphère. Le succès du protocole est mesuré par la charge effective en chlore stratosphérique ou Equivalent Effective Stratospheric Chlorine (EESC), en anglais. Cette quantité est calculée à partir des mesures de CFC, de halons et des autres gaz halogénés au niveau du sol, pondérées par l'efficacité de chaque composé quant à la destruction de l'ozone stratosphérique. La série temporelle obtenue tient compte d'un délai de 3 et 6 ans correspondant au délai moyen de transport des masses d'air vers les régions stratosphériques correspondantes (figure 4). L'évolution de la charge effective en chlore montre que le maximum a été atteint vers la fin des années 1990. À la fin de 2008, 1'EESC avait décru de $11 \%$ par rapport à sa valeur maximale atteinte en 1997. Cette décroissance correspond à $28 \%$ de celle requise pour redescendre au niveau de 1980 , période d'apparition du trou d'ozone polaire. Dans les régions polaires, la décroissance n'était que de $5 \%$ par rapport à la valeur maximale atteinte en 2002. Le temps de résidence très long des principaux CFC dans l'atmosphère (estimé à 100 ans pour le CFC-12) ne permet pas de prévoir un retour avant 2040 aux valeurs qui prévalaient avant l'apparition du trou d'ozone.

\section{Interaction entre la destruction d'ozone stratosphérique et le changement climatique}

La réduction de la charge effective en chlore dans la stratosphère devrait se traduire par une augmentation de l'ozone à l'échelle globale et par une disparition progressive du trou d'ozone en Antarctique. Ce rétablissement de l'ozone mettra plusieurs décennies, le temps que la stratosphère évacue le surplus de composés halogénés introduits par les activités humaines. Cependant, un certain nombre d'incertitudes subsistent quant à la prévision du retour à l'équilibre de l'ozone. Ces incertitudes sont principalement liées au changement climatique qui a pour effet de perturber l'équilibre thermique de la stratosphère. En effet, si l'accroissement de l'abondance des principaux gaz à effet de serre, dont le dioxyde de carbone $\left(\mathrm{CO}_{2}\right)$, a pour effet de réchauffer la troposphère, il refroidit la stratosphère. Ce phénomène est dû au fait que le rayonnement infrarouge terrestre est davantage absorbé dans les basses couches de l'atmosphère et qu'il pénètre moins dans la stratosphère. De plus, dans les conditions de températures stratosphériques, le $\mathrm{CO}_{2}$ émet davantage de rayonnement infrarouge thermique vers l'espace qu'il n'en absorbe.
La réduction de l'ozone stratosphérique induit, elle aussi, un refroidissement de la stratosphère, en raison du rôle très important que joue ce constituant dans l'équilibre thermique de cette région. En effet, l'absorption du rayonnement UV par 1'ozone réchauffe la stratosphère. De l'ordre de $10{ }^{\circ} \mathrm{C}$ par jour vers $50 \mathrm{~km}$, ce réchauffement est compensé en permanence par un refroidissement dû au rayonnement infrarouge du $\mathrm{CO}_{2}$, de la vapeur d'eau et de l'ozone lui-même, dans une moindre mesure. Une diminution de la quantité d'ozone entraîne donc un moindre chauffage et un refroidissement de la stratosphère.

Les analyses des mesures de température par radiosondage et par des instruments satellitaires montrent un refroidissement de 1 à $2{ }^{\circ} \mathrm{C}$, dans la basse stratosphère, et de 4 à $6{ }^{\circ} \mathrm{C}$, dans la haute stratosphère, entre 1980 et 1995, puis une stabilisation des températures par la suite. D'après les modèles, ce refroidissement est principalement causé par la diminution d'ozone (WMO, 2011). Dans la stratosphère polaire, ce refroidissement favorise la formation des nuages stratosphériques polaires et donc les processus de destruction de l'ozone. On a alors un effet de contre-réaction positif par lequel la destruction d'ozone s'auto-entretient en favorisant la formation des nuages stratosphériques polaires. Cet effet a été confirmé par des études qui ont montré que la persistance plus importante du trou d'ozone polaire jusque vers la fin du mois de novembre, observée au cours des années 1990, est directement liée à la destruction de l'ozone. En revanche, dans la haute stratosphère, à l'échelle globale, le refroidissement a un effet inverse puisqu'il ralentit les réactions de destruction de l'ozone et, par effet de contre-réaction négatif, en accélère la reconstitution. Les simulations des modèles climatiques qui utilisent les scénarios du GIEC (Groupe d'experts intergouvernemental sur l'évolution du climat) d'augmentation des gaz à effet de serre et qui incluent le rétablissement de la couche d'ozone prévoient un refroidissement de la stratosphère compris entre 2 et $8^{\circ} \mathrm{C}$ à la fin $\mathrm{du} \mathrm{XXI}$ siècle, majoritairement lié à l'augmentation de la teneur en gaz à effet de serre.

L'étude du rétablissement de la couche d'ozone doit également prendre en compte l'augmentation de l'abondance des gaz sources à partir desquels sont formées les espèces impliquées dans les cycles catalytiques de destruction 
d'ozone. Parmi ceux-ci, la vapeur d'eau joue un rôle particulier puisque c'est le principal gaz source, avec le méthane, des constituants hydrogénés et qu'elle intervient, de plus, dans la formation des nuages stratosphériques polaires. Des mesures à long terme de la vapeur d'eau stratosphérique ont montré un accroissement entre 1980 et 2000, suivi d'une décroissance progressive estimée à 0,5 ppm en 2009. Les mécanismes qui expliquent l'évolution à long terme de la vapeur d'eau stratosphérique restent encore largement incompris. Une augmentation de la vapeur d'eau dans la stratosphère contribuerait à son refroidissement et se répercuterait sur la quantité des composés hydrogénés qui interviennent dans la destruction de l'ozone, vers $20 \mathrm{~km}$ d'altitude. Elle augmenterait aussi la formation des nuages stratosphériques polaires, essentiellement composés d'eau. Dans tous les cas, elle retarderait le rétablissement de l'ozone stratosphérique.

Un troisième paramètre à prendre en compte concerne la propagation des ondes atmosphériques dans la stratosphère. Différentes études ont montré que la propagation de ces ondes permet d'expliquer la circulation méridienne des masses d'air dans la stratosphère, qui se traduit par un mouvement ascendant aux tropiques, suivi par un transport vers les pôles et par un retour vers la troposphère dans les régions polaires (Andrews et al., 1987). Cette circulation, dite de Brewer-Dobson, explique notamment les fortes concentrations d'ozone dans la basse stratosphère des régions polaires, très loin des régions de production situées au-dessus des tropiques où le rayonnement solaire est le plus intense. Le forçage par les ondes de la circulation méridienne stratosphérique est plus important dans l'hémisphère Nord et variable d'une année sur l'autre. L'origine de cette variabilité est encore mal connue. L'évolution de cette circulation méridienne sous l'effet du changement climatique a été simulée par plusieurs modèles climatiques à l'occasion du dernier rapport d'évaluation de la couche d'ozone (WMO, 2011). La plupart des modèles

(1) Le courant-jet subtropical correspond à un maximum de vent zonal dont l'intensité peut atteindre 80 à $100 \mathrm{~m} / \mathrm{s}$ vers $10-12 \mathrm{~km}$ d'altitude. prévoient une intensification de la circulation méridienne qui aurait pour effet d'augmenter le contenu d'ozone dans les régions polaires et de moyenne latitude, accélérant ainsi le rétablissement de l'ozone dans ces régions, et a contrario de le diminuer aux tropiques. Cette intensification de la circulation méridienne n'a pas été encore observée mais sa détection par différents types de mesures fait l'objet d'importantes recherches.

La destruction d'ozone stratosphérique a par ailleurs eu un impact important sur le climat, dans la troposphère et à la surface de la Terre, plus particulièrement dans l'hémisphère Sud où elle est la plus importante. L'effet le plus notable s'est traduit par un déplacement vers le sud du courant-jet troposphérique ${ }^{(1)}$ en été. $\mathrm{Ce}$ déplacement a entraîné une intensification des vents à la surface de l'océan austral et des tendances contrastées de la température de surface estivale en Antarctique, avec un réchauffement au niveau de la péninsule et un refroidissement sur le plateau. D'autres effets moins robustes ont été signalés comme une augmentation de la surface de glace de mer autour du continent antarctique, un déplacement vers le sud du train de dépression dans l'hémisphère Sud et des précipitations associées, et une décroissance du puits de carbone dans l'océan austral (WMO, 2011).

\section{État actuel de la couche d'ozone et prévisions pour son rétablissement}

Depuis une dizaine d'années, le contenu intégré d'ozone s'est stabilisé à l'échelle globale et il montre même des signes d'accroissement dans certaines régions de l'hémisphère Nord. Le dernier rapport d'évaluation de la couche d'ozone fait état de valeurs moyennes de contenu intégré inférieures d'environ 3,5\% aux valeurs mesurées avant 1980, dans les régions comprises entre $60^{\circ} \mathrm{N}$ et $60^{\circ} \mathrm{S}$. Aux moyennes latitudes, le contenu intégré d'ozone a été réduit d'environ $6 \%$ dans l'hémisphère Sud et de $3 \%$ dans l'hémisphère Nord, avec une diminution négligeable au-dessus des tropiques (figure 5). Une stabilisation similaire a été observée sur la distribution verticale d'ozone, tant dans la basse que dans la haute stratosphère (WMO, 2011).

Figure 5 - Déviations du contenu intégré d'ozone, en moyenne annuelle, par rapport à la moyenne de la période 1964-1980, dans différentes bandes de latitude $60^{\circ} \mathrm{S}-60^{\circ} \mathrm{N}, 90^{\circ} \mathrm{S}-90^{\circ} \mathrm{N}, 35^{\circ} \mathrm{N}-60^{\circ} \mathrm{N}, 35^{\circ} \mathrm{S}$ $\left.60^{\circ} \mathrm{S}, 25^{\circ} \mathrm{S}-25^{\circ} \mathrm{N}\right)$, estimée à partir de mesures au sol (courbes noires) et de différentes séries de mesures satellitaires. (Adapté de WMO, 2011.)
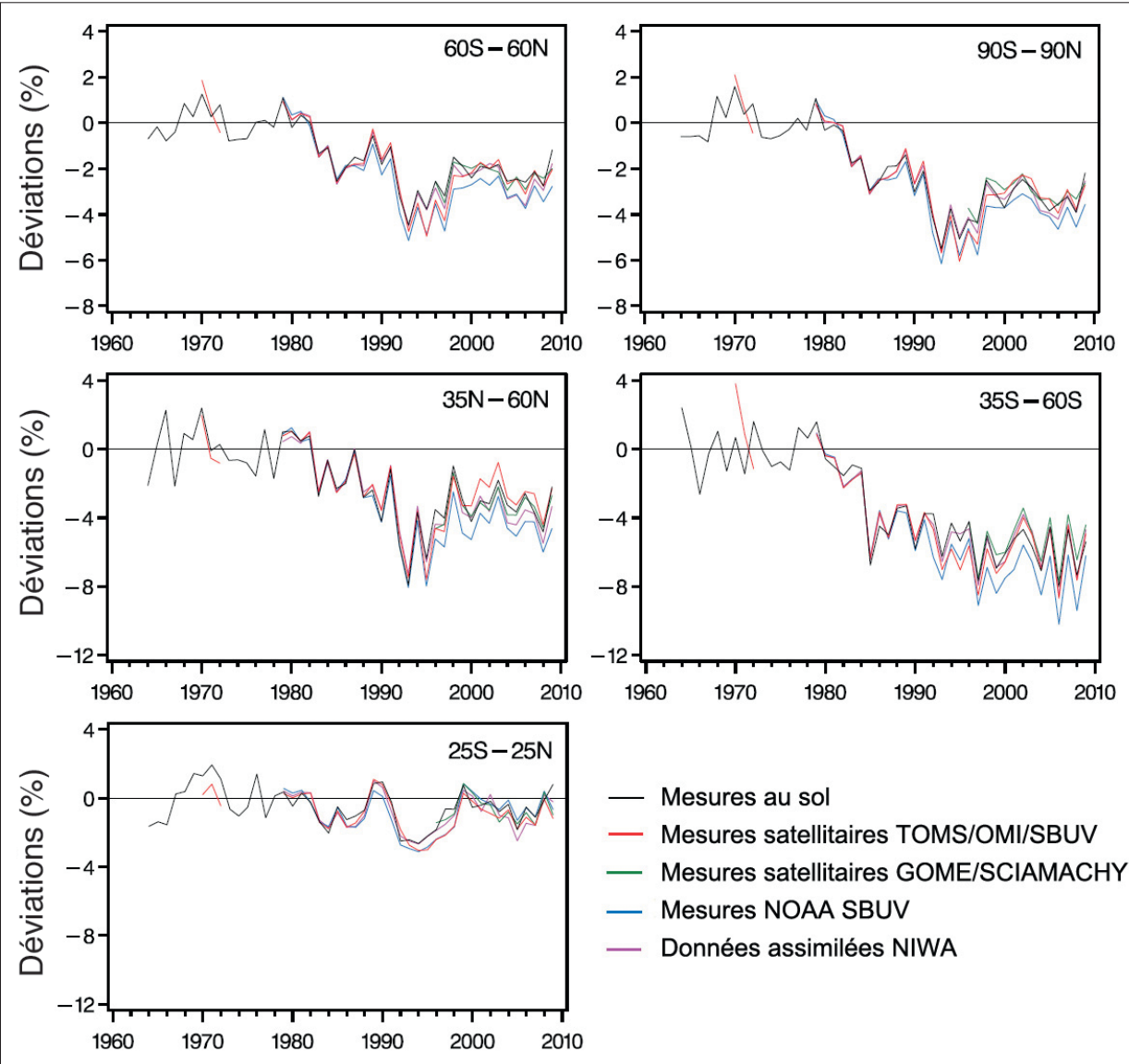


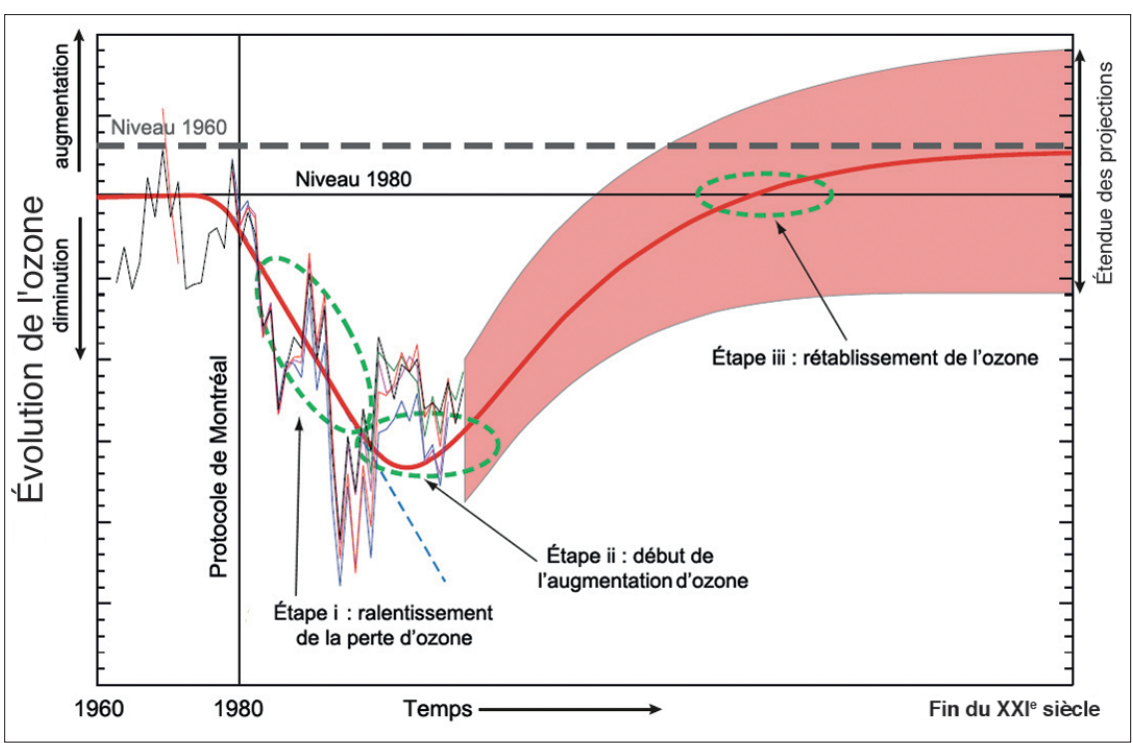

Figure 6 - Schéma d'évolution du contenu intégré d'ozone entre $60^{\circ} \mathrm{N}$ et $60^{\circ} \mathrm{S}$, sur la période 1960-2100. Les courbes en couleur représentent les différentes observations d'ozone au sol et satellitaires. La courbe rouge en trait gras illustre l'évolution de l'ozone passée et simulée dans le futur. La zone rose représente l'étendue des projections des modèles. La ligne noire en trait plein montre le niveau des années 1980, pris comme référence pour le protocole de Montréal. La ligne noire en tirets indique les niveaux très incertains des années 1960. Les trois étapes de rétablissement de la couche d'ozone sont indiquées par les ellipses vertes en tirets. (Adapté de WMO, 2011).

Les modèles de climat indiquent que les valeurs minimales d'ozone ont été atteintes dans les régions polaires et aux moyennes latitudes en ce début du XXI ${ }^{\mathrm{e}}$ siècle. Ils prévoient pratiquement tous une accélération du retour à l'équilibre de l'ozone, du fait de l'intensification de la circulation méridienne liée à l'augmentation de la teneur en gaz à effet de serre dans l'atmosphère. Le rétablissement de la couche d'ozone est ainsi prévu dans une période de temps comprise entre 2015 et 2030 , dans 1'hémisphère Nord, et entre 2030 et 2040, dans l'hémisphère Sud. La disparition du trou d'ozone ne devrait intervenir qu'en dernier lieu, après 2050. Du fait de l'accélération de la circulation méridienne et de la baisse de température vers $40 \mathrm{~km}$, les modèles prévoient également un sur-rétablissement (superrecovery en anglais) de l'ozone aux moyennes latitudes et dans les régions polaires, et un sous-rétablissement (under-recovery) au-dessus des tropiques. Les différentes étapes de la reconstitution de la couche d'ozone sont illustrées dans la figure 6 .

\section{Double bénéfice du protocole de Montréal}

Outre son action sur les substances destructrices d'ozone, il est important de souligner que le protocole de Montréal a aussi contribué de façon importante à la lutte contre l'effet de serre additionnel lié aux activités humaines. En effet, leur potentiel de réchauffement comparé à celui du $\mathrm{CO}_{2}$, on peut exprimer ces émissions en émissions équivalentes de $\mathrm{CO}_{2}$. Une étude a ainsi montré que l'application, entre 1990 et 2010, du protocole de Montréal a été équivalente à une réduction d'environ 8 milliards de tonnes de dioxyde de carbone par an. Cette réduction est à mettre en perspective avec la réduction d'émission de 2 milliards de tonnes de $\mathrm{CO}_{2}$ par an préconisée dans le cadre du protocole de Kyoto sur la même période (Velders et al., 2007). À titre de comparaison également, les émissions anthropiques de $\mathrm{CO}_{2}$ étaient de 34 milliards de tonnes en 2011.

Compte tenu de la synergie entre les protocoles de Montréal et Kyoto, un nouvel amendement au protocole de Montréal a été signé en 2007, qui prévoit d'accélérer l'arrêt des émissions de HCFC. Dans les pays développés, la production sera gelée en 2013 puis réduite progressivement, jusqu'à une élimination complète en 2030, tandis que les autres pays (dits de l'article $5 \mathrm{du}$ protocole de Montrréal) se sont mis d'accord pour un arrêt de la production des HCFC en 2040. Avec l'élimination des HCFC, la demande en production de HFC devrait croître substantiellement. Or ceux-ci sont également des gaz à effet de serre. Il est donc

Figure 7 - Indice UV simulé à midi, en juillet, aux moyennes latitudes de l'hémisphère Nord, selon différents scénarios : en l'absence de protocole (courbe noire), suivant le protocole de Montréal de réduction des substances destructrices d'ozone (courbe rouge), pour une valeur fixe d'EESC correspondant aux niveaux de 1960 (courbe verte), et aux niveaux moyens entre 1975 et 1985 (courbe grise). L'échelle de couleur habituellement utilisée pour indiquer la dangerosité du rayonnement UV en surface est également indiquée. (Adapté de Newman et al., 2009.)

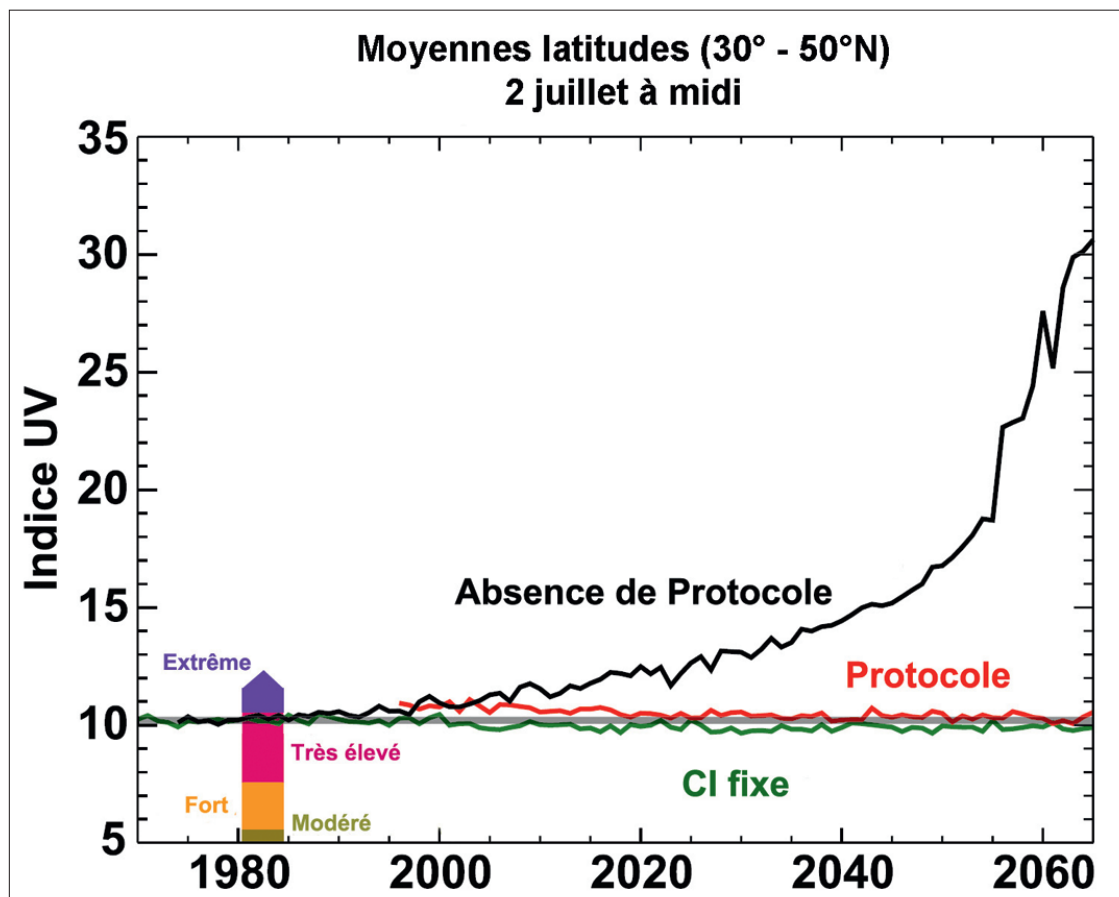


important de promouvoir la production de HFC à faible potentiel de réchauffement. De nouveaux fluorocarbures ont ainsi été proposés, comme le HFC1234yf, pour des applications de climatisation, dont le potentiel de réchauffement à 100 ans est de 4 . Pour tout nouveau substitut proposé, il est toutefois important de bien en évaluer la toxicité à la production et les conséquences pour l'environnement, notamment en ce qui concerne les produits de décomposition.

\section{Conclusion}

Le protocole de Montréal a atteint son premier objectif de réduction de la charge en constituants halogénés nocifs pour la couche d'ozone, tout en fournissant un co-bénéfice substantiel en termes de réduction d'émission de gaz à effet de serre. Des simulations ont montré qu'en l'absence de protocole, la charge totale en chlore stratosphérique aurait dépassé largement le maximum de 3,5 ppbv pour atteindre 45 ppbv en
2065 avec des conséquences dramatiques pour la couche d'ozone telles que l'apparition d'un trou d'ozone en Arctique en 2020 et des teneurs globales en ozone inférieures, en 2065, au tiers de celles qui prévalaient dans les années 1970 (Newman et al., 2009). Le rayonnement UV de surface aurait alors atteint des niveaux inédits et potentiellement très dangereux (figure 7).

Si la diminution du contenu atmosphérique en substances destructrices d'ozone est avérée, l'efficacité du protocole quant au rétablissement de l'ozone stratosphérique doit maintenant être démontrée. À cet égard, une première étape a été franchie avec la stabilisation du contenu intégré et de la distribution verticale d'ozone à l'échelle globale depuis 2000. L'enjeu est maintenant de détecter l'augmentation de ces paramètres. Or la variabilité atmosphérique, plus ou moins importante selon les régions, peut brouiller le signal, comme en témoigne la destruction record d'ozone en Arctique au printemps 2011. Malgré certains signes d'accroissement des teneurs en ozone dans l'hémisphère Nord, le dernier rapport d'évaluation de la couche d'ozone ne concluait pas à une augmentation significative de l'ozone imputable directement à la diminution du contenu en composés halogénés stratosphériques (WMO, 2011). Depuis le dernier rapport d'évaluation, une étude a été publiée qui montre les premiers signes d'augmentation du contenu intégré d'ozone en Antarctique, au mois d'octobre, indépendante de la variabilité atmosphérique (Salby et al., 2011). Cependant, cette étude doit être confirmée et complétée par des résultats dans d'autres régions. Les signes indiscutables du rétablissement de l'ozone stratosphérique seront notamment une diminution persistante de la taille du trou d'ozone antarctique et, en parallèle, une augmentation des niveaux d'ozone stratosphérique à l'échelle globale. Il est donc indispensable de continuer à surveiller avec attention l'abondance de ce gaz si indispensable à la vie sur Terre.

\section{Bibliographie}

\footnotetext{
Allen D. R., R. M. Bevilacqua, G. E. Nedoluha, C. R. Randall et G. L. Manney, 2003 : Unusual stratospheric transport and mixing during the 2002 Antarctic winter. Geophys. Res. Lett., 30, 1599, doi:10.1029/2003GL017117.

Andrews D. G., J. R. Holton et C. B. Leovy, 1987 : Middle Atmosphere Dynamics. Academic Press, International Geophysics series, vol. 40.

Chubachi S., 1984 : A special ozone observation at Syowa station, Antarctica, from February 1982 to January 1983. Dans : Quadrennial Ozone Symposium of the International Ozone Commission, C. S. Zerefos et A. M. Chazi, Eds., Reidel Dordrecht, Halkidiki, Greece, p. 285-289.

Farman J. C., B. J. Gardiner et J. D. Shanklin, 1985 : Large losses of total ozone in Antarctica reveal seasonal CLOX/NOX interaction. Nature, 315, 207-210.

Manney G. L., M. L. Santee, M. Rex, N. J. Livesey, M. C. Pitts, P. Veefkind, E. R. Nash, I. Wohltmann, R. Lehmann, L. Froidevaux, L. R. Poole, M. R. Schoeberl, D. P. Haffner, J. Davies, V. Dorokhov, H. Gernandt, B. Johnson, R. Kivi, E. Kyrö, N. Larsen, P. F. Levelt, A. Makshtas, C. T. McElroy, H. Nakajima, M. C. Parrondo, D. W. Tarasick, P. von der Gathen, K. A. Walker et N. S. Zinoviev, 2011 : Unprecedented Arctic 0zone loss in 2011. Nature, 478, 469-475, doi:10.1038/nature10556

Newman P. A., L. D. Oman, A. R. Douglass, E. L. Fleming, S. M. Frith, M. M. Hurwitz, S. R. Kawa, C. H. Jackman, N. A. Krotkov, E. R. Nash, J. E. Nielsen,

S. Pawson, R. S. Stolarski et G. J. M. Velders, 2009 : What would have happened to the ozone layer if chlorofluorocarbons (CFCs) had not been regulated? Atmos. Chem. Phys., 9, 2113-2128, doi:10.5194/acp-9-2113-2009

Salby M., E. Titova et L. Deschamps, 2011 : Rebound of Antarctic 0zone. Geophys. Res. Lett., 38, L09702, doi:10.1029/2011GL047266.

Solomon S., R. R. Garcia, F. S. Rowland et D. J. Wuebbles, 1986 : On the depletion of Antarctic ozone. Nature, 321, 755-758.

Velders G. J. M., S. O. Andersen, J. S. Daniel, D. W. Fahey et M. McFarland, 2007 : The importance of the Montreal Protocol in protecting climate. Proc. Natl. Acad. Sci., 104(12), 4814-4819, doi:10.1073/pnas.0610328104

WMO, 1985 : Report 16, Atmospheric Ozone. Global Ozone Research and Monitoring Project, Geneva.

WMO, 1998 : Report 43, Assessment of trends in the vertical distribution of ozone. Global Ozone research and monitoring project, Geneva.

WMO, 2011 : Report 53, Scientific assessment of ozone depletion 2010. Global ozone research and monitoring project, Geneva.
} 